\title{
Triplet Excitation and Electroluminescence from a Supramolecular Monolayer Embedded in a Boron Nitride Tunnel Barrier
}

\author{
Simon A. Svatek, ${ }^{*},, \# \oplus$ James Kerfoot, ${ }^{\dagger}, \#$ Alex Summerfield, ${ }^{\dagger}$ Anton S. Nizovtsev, ${ }^{\ddagger}, \S$ \\ Vladimir V. Korolkov, ${ }^{\dagger}$ Takashi Taniguchi, ${ }^{\|}$Kenji Watanabe, ${ }^{\|}$Elisa Antolín, ${ }^{\perp}$ Elena Besley, ${ }^{\ddagger \odot}$ \\ and Peter H. Beton $*{ }^{*} \odot$ \\ ${ }^{\dagger}$ School of Physics and Astronomy and ${ }^{\ddagger}$ School of Chemistry, University of Nottingham, Nottingham NG7 2RD, U.K. \\ ${ }^{\S}$ Nikolaev Institute of Inorganic Chemistry, Siberian Branch of the Russian Academy of Sciences, Academician Lavrentiev Avenue 3, \\ Novosibirsk 630090, Russian Federation \\ "National Institute for Materials Science, 1-1 Namiki, Tsukuba 305-0044, Ibaraki, Japan \\ ${ }^{\perp}$ Instituto de Energía Solar, Universidad Politécnica de Madrid, Avenida Complutense 30, Madrid 28040, Spain
}

Supporting Information

\begin{abstract}
We show that ordered monolayers of organic molecules stabilized by hydrogen bonding on the surface of exfoliated few-layer hexagonal boron nitride ( $\mathrm{hBN}$ ) flakes may be incorporated into van der Waals heterostructures with integral few-layer graphene contacts forming a molecular/twodimensional hybrid tunneling diode. Electrons can tunnel through the hBN/molecular barrier under an applied voltage $V_{\mathrm{SD}}$, and we observe molecular electroluminescence from an excited singlet state with an emitted photon energy $h \nu>e V_{\mathrm{SD}}$, indicating upconversion by energies up to $\sim 1 \mathrm{eV}$. We show that tunneling electrons excite embedded molecules into singlet states in a two-step process via an intermediate triplet

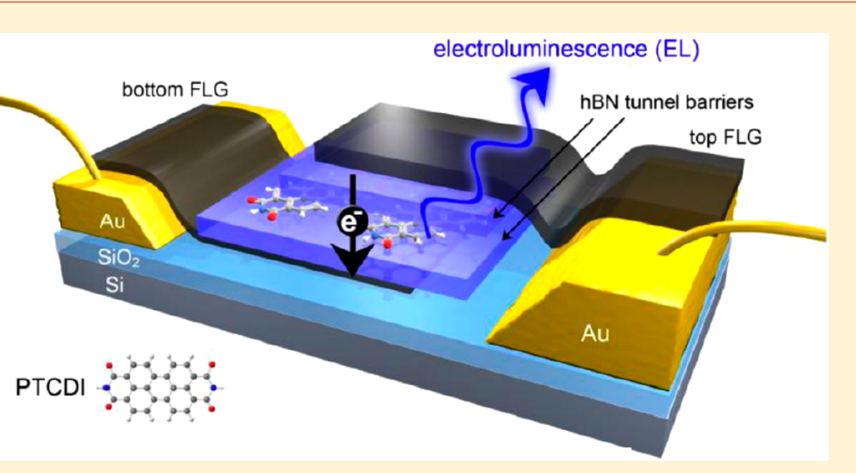
state through inelastic scattering and also observe direct emission from the triplet state. These heterostructures provide a solidstate device in which spin-triplet states, which cannot be generated by optical transitions, can be controllably excited and provide a new route to investigate the physics, chemistry, and quantum spin-based applications of triplet generation, emission, and molecular photon upconversion.
\end{abstract}

KEYWORDS: Upconversion, molecular self-assembly, triplets, van der Waals heterostructures, molecular electronics, tunnel diodes

$\mathrm{T}$ wo-dimensional supramolecular arrays stabilized by noncovalent interactions provide a highly flexible route to the spatial organization, down to the molecular scale, of functional molecules on a surface. ${ }^{1-4}$ While this route to surface patterning has been successful in positioning chemical groups within adsorbed monolayers, the noncovalent nature of the stabilizing interactions has limited the possibilities to explore the transport of charge through the component molecules. One possible route to explore the electrical properties of supramolecular monolayers is through the use of adjacent charge injection layers placed above and/or below the molecules. This architecture, in which current flows perpendicular to the plane of the adsorbed molecules, would require that the supramolecular layer is embedded in a more complex heterostructure with integral contact and spacer layers. Although there has been recent progress in the growth of all-organic epitaxial supramolecular heterojunctions, ${ }^{5-7}$ these structures cannot currently be prepared with the required complexity and control. The techniques used to fabricate van der Waals heterostructures, ${ }^{8}$ such as a tunnel diode formed by placing few-layer hexagonal boron nitride (hBN) between two graphene layers, ${ }^{9}$ offer an alternative approach. In this Letter we show that a similar device architecture may be employed to embed a supramolecular monolayer between two hBN tunnel barriers, thus forming a hybrid molecular/two-dimensional (2D) device. The encapsulated organic molecules can be excited electrically and subsequently relax through the emission of photons resulting in electroluminescence (EL) from both singlet and spin-triplet states. Photons are upconverted by energies up to $1 \mathrm{eV}$, and we show that singlets are excited through a multi-electron inelastic process via a triplet intermediate state. This hybrid structure provides a solid-state device in which triplets can be controllably excited, and it offers a route to fundamental studies of long-lived excitations with non-zero spin and their relevance to lowvoltage light-emitting devices and quantum spin-based excitonic and electronic devices.

Received: September 13, 2019

Revised: November 28, 2019

Published: December 10, 2019 

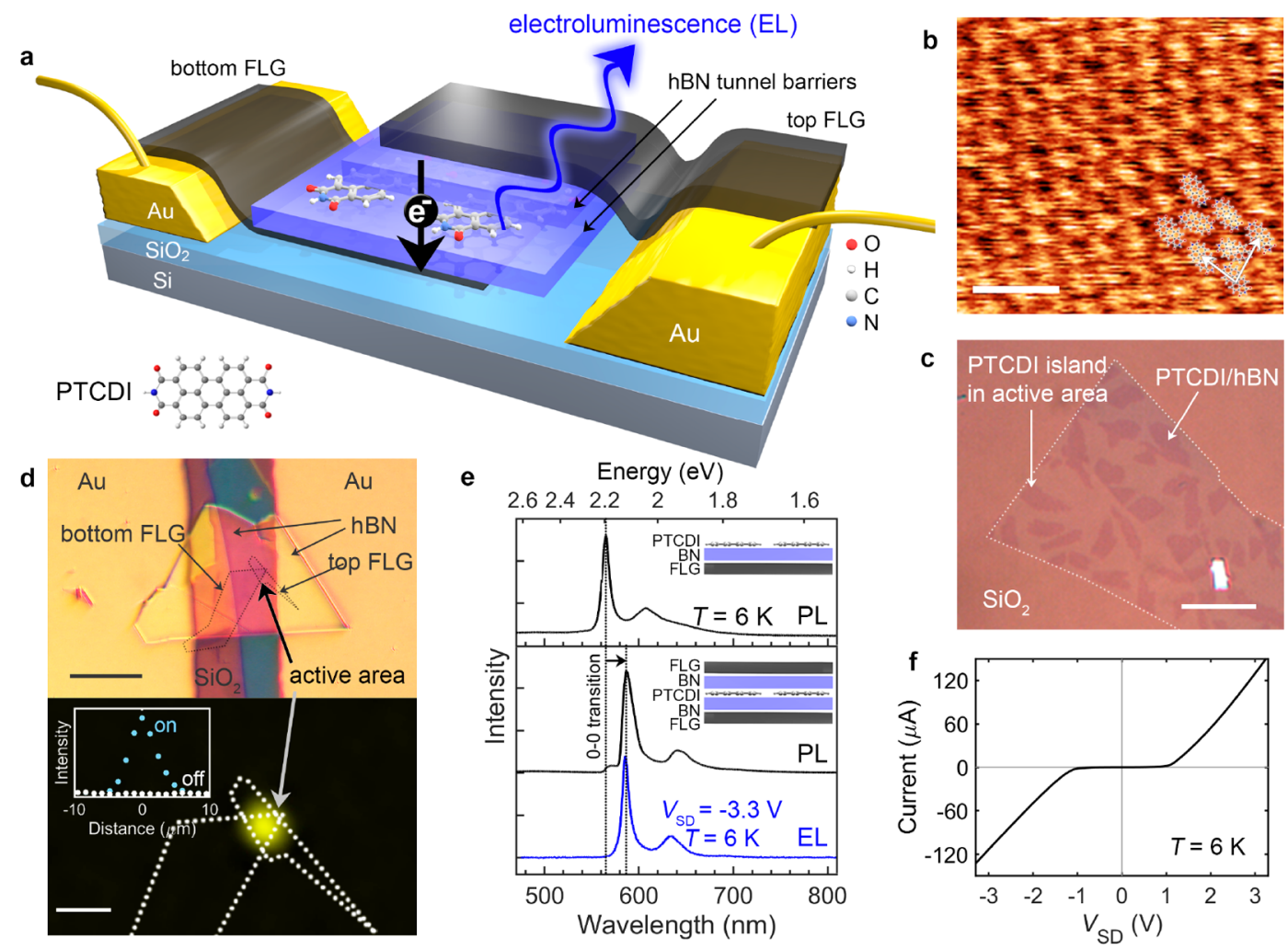

Figure 1. FLG/BN/PTCDI/hBN/FLG heterostructures. (a) Schematic of a device in which a monolayer of PTCDI is encapsulated between two hBN tunnel barriers, and charge is injected from upper and lower FLG contacts (the upper thick hBN layer and a supporting thick hBN flake, which provides a supporting substrate for the lower graphene, are omitted for clarity; neither plays an active role in the device operation); (lower left) schematic of the molecular structure of PTCDI. (b) AFM image of a monolayer-thick island of PTCDI on hBN; the lattice vectors of the molecular array are marked and have the following values: $1.48 \pm 0.1$ and $1.78 \pm 0.1 \mathrm{~nm}$, subtended by an angle of $89^{\circ}$. (c) Optical micrograph showing monolayer islands following sublimation of 0.5 monolayers of PTCDI on a bilayer hBN flake (highlighted by dotted outline); this flake forms the lower tunnel barrier for this device, and the island selected for the active region is marked by an arrow-note that the PTCDI grows in a different morphology on the surrounding $\mathrm{SiO}_{2}$ surface and cannot be resolved in these regions. (d, upper) Optical image of a device showing gold contacts and van der Waals heterostructure. The active area of the device where the upper and lower FLG layers overlap is highlighted; (d, lower) optical image of the device taken under bias $\left(V_{S D}=-3.2 \mathrm{~V}\right)$ acquired with an exposure time of $8 \mathrm{~s}$ with an overlay also showing the position of the graphene contacts and confirming that light is emitted from the active area of the device; (inset) the variation of intensity along the horizontal axis through (on) and away (off) the active region. (e) EL (acquisition time $100 \mathrm{~s}$ ) and PL spectra of device acquired at liquid helium temperatures; the PL for an uncapped device is also included highlighting the peak shift due to encapsulation. (f) Current-voltage characteristics. Scale bars (b) 3 $\mathrm{nm}$, (c) $20 \mu \mathrm{m}$, (d) $20 \mu \mathrm{m}$ (upper), and $6 \mu \mathrm{m}$ (lower).

To fabricate our devices, we use polymer stamp-assisted van der Waals assembly ${ }^{10-12}$ to sequentially pick up flakes of fewlayer graphene (FLG) and hBN. hBN flakes with adsorbed monolayers of organic molecules can be picked up and deposited as part of the assembly process in the same way as pristine flakes, thus allowing the integration of molecular layers within van der Waals heterostructures. We first use a polymer "stamp" to pick up a large hBN flake (lateral dimensions 10s of $\mu \mathrm{m}$, thickness $10 \mathrm{~s}$ of $\mathrm{nm}$ ), which is ultimately used to cap the device. This hBN flake is then used to pick up an FLG flake, which serves as the top contact, followed by a thin $(\leq 1 \mathrm{~nm})$ hBN flake, which forms the upper tunnel barrier. The van der Waals stack is then used to pick up a second hBN tunnel barrier, also with a thickness of 1-3 monolayers, on which a molecular monolayer has been deposited by sublimation. This part-formed tunneling device is then released from the stamp onto a second FLG flake, which forms the lower contact; the release site is chosen so that the upper and lower FLG layers make independent contact with two preformed contacts (10 $\mathrm{nm} \mathrm{Cr} / 30 \mathrm{~nm} \mathrm{Au}$ ). Further details are provided in the Supporting Information.
A schematic of a completed device with an embedded monolayer of sublimed perylene tetracarboxylic di-imide (PTCDI) is shown in Figure 1a. The FLG provides semitransparent top- and bottom-electrodes, and the hBN layers allow carrier injection via tunnelling under an applied bias, while suppressing quenching from the FLG contacts. ${ }^{13}$ PTCDI is a planar molecule and is adsorbed parallel to the hBN substrate in $2 \mathrm{D}$ islands stabilized by hydrogen bonding. ${ }^{14}$ The islands have monolayer height and typical lateral dimensions of 5-10 $\mu \mathrm{m}$, and for the deposition parameters we use (see Supporting Information) a surface coverage of $50 \%$. The molecular ordering within the islands is resolved using atomic force microscopy (AFM), which reveals lattice vectors close to the expected value ${ }^{14}$ (see Figure $1 \mathrm{~b}$ ). At a large scale, individual monolayer PTCDI islands may be identified using optical microscopy during the transfer process (Figure 1c). Images of a completed device are shown in Figure $1 \mathrm{~d}$; the active area of the device (where the upper and lower FLG layers overlap) is marked. It is possible to selectively encapsulate a selected monolayer-height PTCDI island in the active region; for this device we used the island highlighted by an arrow in Figure 1c, which had been adsorbed on a bilayer 

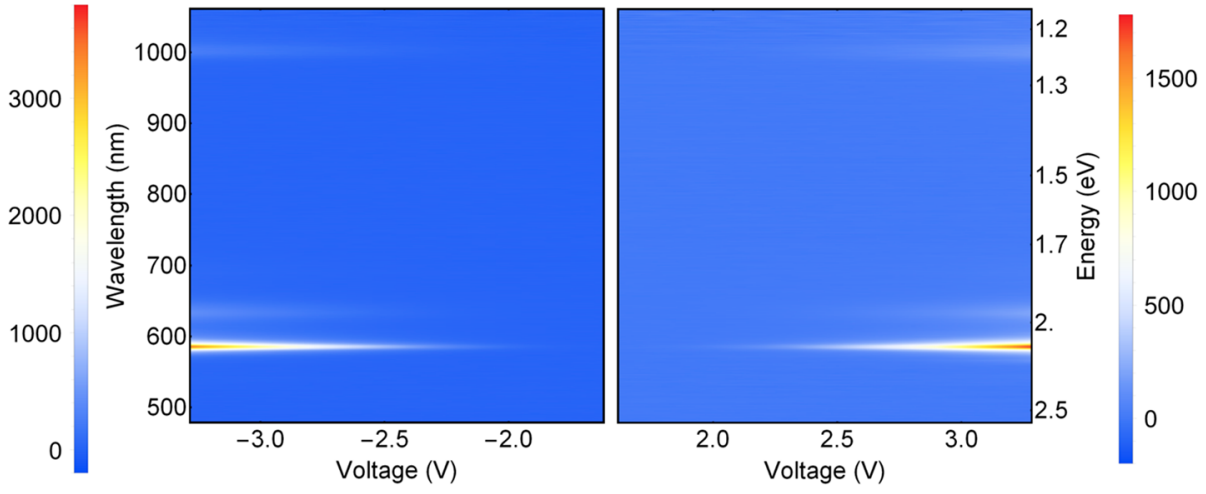

Figure 2. Voltage map of EL. The EL intensity is displayed as a color map for different voltages (horizontal axis) and wavelengths (vertical axis). These data show that the EL peak position is constant over the measured voltage range.

hBN flake, which forms the lower tunnel barrier. The effective area of the device is estimated to be $4 \mu \mathrm{m}^{2}$. The deposition of PTCDI, and the preparation of the hBN surface, is discussed in the Supporting Information.

The current-voltage characteristics of the device were measured in an optical cryostat at a temperature $T=6 \pm 1 \mathrm{~K}$, and they are highly nonlinear as expected for a tunneling device (see Figure 1f). The devices emit light when a current flows. The EL spectrum acquired for an applied bias $V_{\mathrm{SD}}=$ $-3.3 \mathrm{~V}$ (Figure 1e) shows an intense peak at the wavelength of $586.1 \pm 0.5 \mathrm{~nm}(2.115 \pm 0.002 \mathrm{eV})$ corresponding to the zero phonon transition $(0-0)$ from the lowest excited singlet, $S_{1}$, to the ground state, $S_{0}$, accompanied by a satellite vibronic $0-1$ peak $(633.4 \pm 0.5 \mathrm{~nm} / 1.958 \pm 0.002 \mathrm{eV})$. An optical image of the device under bias confirms that the active region of the device is the source of the photon emission (Figure 1d). EL emission is observed in both polarities, and the peak position varies by less than $0.5 \mathrm{~nm}$ over the measured voltage range (see Figure 2).

The EL peaks are close to the $0-0$ transition $(588.1 \pm 0.5$ $\mathrm{nm} / 2.108 \pm 0.002 \mathrm{eV})$ in the photoluminescence (PL) spectrum of the device (Figure 1e). However, both the PL and EL peaks are shifted from the $0-0$ transition $(565.0 \pm 0.5$ $\mathrm{nm} / 2.194 \pm 0.002 \mathrm{eV}$ ) measured for uncapped PTCDI (also shown in Figure 1e) on a single hBN layer by $0.086 \pm 0.003$ $\mathrm{eV}$ (EL and PL spectra are also readily acquired at room temperature; the PL peak positions are independent of voltage-see Supporting Information). We previously showed that, for uncapped PTCDI on hBN, a combination of resonant and nonresonant interactions between adsorbed PTCDI and $\mathrm{hBN}$ leads to a red shift of $0.31 \mathrm{eV}$ compared with gas-phase spectra. ${ }^{15,16}$ A further red shift is expected when a second hBN layer is added in the encapsulation process due to the additional changes in dielectric environment. This is discussed in more detail in Supporting Information, where we calculate the transition energies of uncapped $(2.26 \mathrm{eV})$ and capped $(2.20 \mathrm{eV})$ PTCDI and, in particular, a predicted red shift of $0.06 \mathrm{eV}$, which is due to the addition of a second hBN layer; these values are in good agreement with our experimental values.

The EL spectrum of PTCDI exhibits significant upconversion, that is emission of photons with energies $h \nu>e V_{\mathrm{SD}}$, the energy gained by a tunneling electron when passing ballistically between the two contacts. ${ }^{17}$ This implies that the emission must occur through a multielectron process, rather than through simple charge injection into the highest occupied molecular orbital (HOMO) and lowest unoccupied molecular orbital (LUMO) levels of the molecule (as might be expected for a molecular analogue of a van der Waals heterostructure with a transition-metal dichalcogenide emissive layer ${ }^{13}$ ), and is suggestive of an inelastic scattering mechanism. Figure 3a shows spectra acquired in this voltage range, and we see a significant intensity in the $0-0$ peak down to $\left|V_{\mathrm{SD}}\right|=1.6 \mathrm{~V}$ and
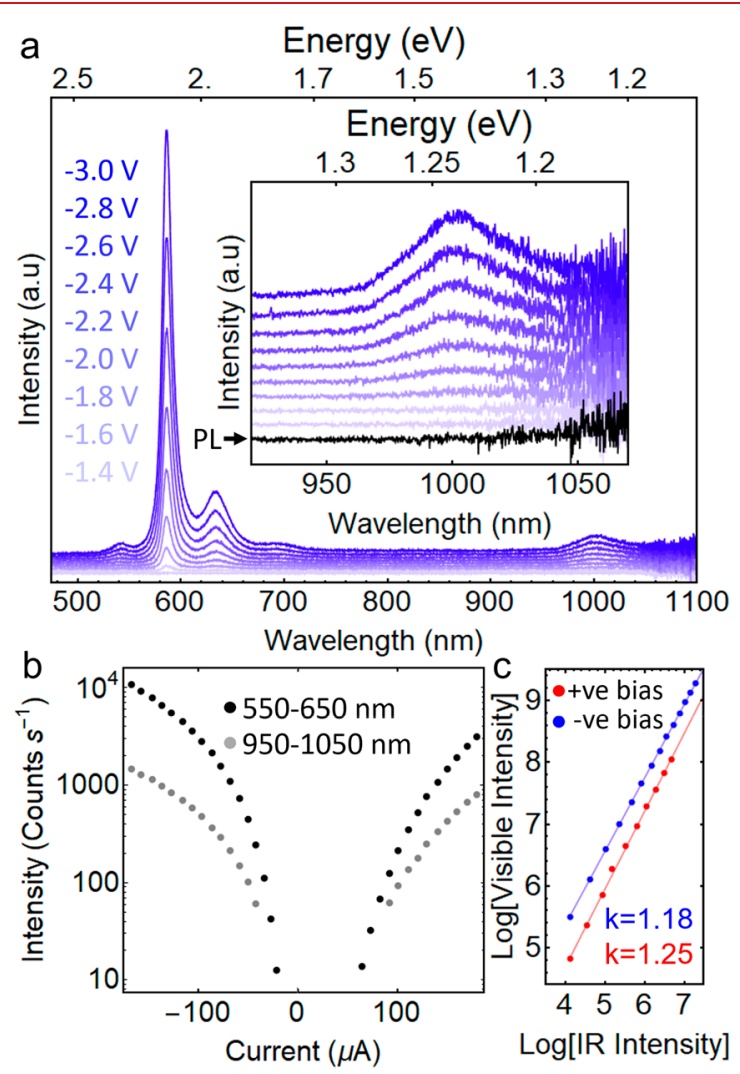

Figure 3. Photon upconversion and triplet EL from a FLG/BN/ PTCDI/hBN/FLG heterostructure. (a) Spectra acquired at $6 \mathrm{~K}$ with a $100 \mathrm{~s}$ integration time for a series of applied voltages ranging from -1.4 to $-3.0 \mathrm{~V}$; (inset) EL in the infrared plotted together with the PL spectrum in the same spectral region (black). (b) The EL signal (with background subtracted) integrated between 550 and $650 \mathrm{~nm}$ and between 950 and $1050 \mathrm{~nm}$, vs current. (c) Logarithmic plot of the integrated intensity between 550 and $650 \mathrm{~nm}$ vs. the intensity between 950 and $1050 \mathrm{~nm}$ showing a near-linear dependence between the singlet and triplet emission intensities. 
at room temperature (see Supporting Information) down to $1.3 \mathrm{~V}$, implying an upconversion energy much greater than $k_{\mathrm{B}} T$ (ruling out thermal effects) and close to $1 \mathrm{eV}$. In this device we also resolve an additional weak peak at $543.8 \pm 0.5 \mathrm{~nm}(2.280$ $\pm 0.002 \mathrm{eV})$. We tentatively assign this feature to a $1-0$ vibronic transition, although the possible origin of a PTCDI in a different trapped conformation cannot be ruled out.

The observation of photon upconversion indicates that molecules are excited into an intermediate state and then, through a further excitation, are excited into the singlet excited state $S_{1}$. EL is observed in this device for a current density down to $\sim 1 \mathrm{pA} \mathrm{nm}^{-2}$, which corresponds, approximately, to an average time between the traversal of electrons through each molecule of order $0.1 \mu \mathrm{s}$ (assuming $\sim 1 \mathrm{~nm}^{2} /$ molecule). The relevant excitation for the above-threshold emission must have a lifetime of this order of magnitude (or longer) thus ruling out mechanisms such as the sequential excitation of vibronic modes of the $S_{0}$ electronic ground state, which relax on a much more rapid time scale. In common with Chen et al. ${ }^{18}$ we suggest that the long lifetime strongly suggests that the intermediate state involved in photon upconversion is a $T_{1}$ spin-triplet.

The role of triplets is confirmed through the identification of an additional peak in the EL spectrum at $\sim 1004 \pm 1 \mathrm{~nm}$, photon energy $1.235 \pm 0.001 \mathrm{eV}$. This peak is not present in the PL spectrum (see Figure 3a inset), but it is close to the value, $1.18 \mathrm{eV}$, of the triplet state of a related PTCDI derivative determined $^{19}$ by triplet pair absorption. Accordingly we attribute this peak to emission from the $T_{1}$ state. The observed energy is also close to the energy, $1.29 \mathrm{eV}$, calculated for this transition (see Supporting Information).

Figure $3 \mathrm{~b}$ shows that the emission intensities $I\left(\mathrm{~T}_{1}\right)$ and $I\left(S_{1}\right)$ from, respectively, the $T_{1}$ and $S_{1}$ states have a highly nonlinear dependence on current. A logarithmic plot (Figure $3 \mathrm{c}$ ) shows a power law dependence of $I\left(\mathrm{~S}_{1}\right)$ on $I\left(\mathrm{~T}_{1}\right)$ over a large voltage range in both forward and reverse bias, $I\left(\mathrm{~S}_{1}\right) \propto$ $I\left(\mathrm{~T}_{1}\right)^{k}$, where $k \approx 1.2$ indicating a near linear relationship. Assuming that, for a given applied voltage, the number of excited triplets is proportional to the $T_{1}$ intensity, triplettriplet annihilation (TTA) ${ }^{20}$ may be ruled out as a route to the secondary excitation of molecules from $T_{1}$ to $S_{1}$, since for this mechanism a quadratic dependence ${ }^{21}(k \geq 2)$ on the number of triplets would be expected (assuming a low density of triplets, ${ }^{22}$ which is likely for the low currents we observe close to the threshold for emission). We therefore suggest that the $\mathrm{T}_{1}$ to $\mathrm{S}_{1}$ transition is promoted by a second inelastic electronscattering event.

The proposed mechanism is summarized in the band diagrams shown in Figure 4a,b. No emission is expected until the energy gained by tunneling electrons, $\mathrm{e} V_{\mathrm{SD}}$, exceeds the energy difference between the $S_{0}$ and $T_{1}$ states thus permitting excitation between these states through an inelastic process. We also see evidence for inelastic scattering in the electrical characteristics of the device. Figure $4 \mathrm{c}$ shows that a broad peak is observed in the second derivative of $\mathrm{d}^{2} I / \mathrm{d} V^{2}$ at $\sim 1.1 \mathrm{~V}$, very close to the triplet energy. Peaks in $\mathrm{d}^{2} I / \mathrm{d} V^{2}$ due to inelastic electron scattering are expected when the voltage drop matches the energy of an excitation to which electrons are coupled ${ }^{23,24}$ (similar features in $\mathrm{hBN} /$ graphene tunnel devices are observed to inelastic scattering of phonons ${ }^{25}$ ). Molecules in the $\mathrm{T}_{1}$ state may undergo a further inelastic excitation to the $S_{1}$ state or relax via the emission of a photon. This simple model is consistent with the voltage thresholds, peak energies, and
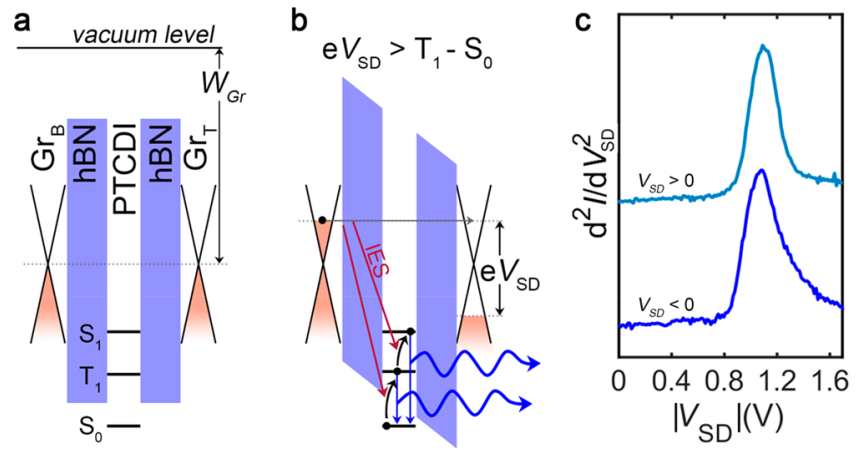

Figure 4. Mechanism and inelastic tunneling spectroscopy. (a) Alignment of work function of graphene $\left(W_{\mathrm{Gr}}\right), \mathrm{hBN}$ bands, and molecular energy levels under zero bias. (b) Band alignment under bias $V_{\mathrm{SD}}$; molecule can be inelastically excited when $\mathrm{e} V_{\mathrm{SD}}$ exceeds the energy difference between the $S_{0}$ and $T_{1}$ states. Molecule undergoes a further inelastic excitation to the $S_{1}$ state; photons can be emitted from a transition to $S_{0}$ from either the $S_{1}$ or $T_{1}$ states. (c) Peaks in $\mathrm{d}^{2} I / \mathrm{d} V^{2}$ occur where $\mathrm{e} V_{\mathrm{SD}}$ matches the energy of the excitation. Clear peaks are observed for $V_{\mathrm{SD}} \approx 1.1 \mathrm{~V}$ close to the value expected for triplet excitation via inelastic scattering.

variation of intensity ratio, which we observe, and it implies that the inelastic scattering process induces a change in the spin state of the molecule.

The generation of excitons in our devices shows fundamental differences to the mechanism in conventional organic light-emitting diodes (OLEDs), where electrons and holes are injected from remote contacts and combine to form both singlet and triplet excitons. The spin degeneracy of these states typically leads ${ }^{26}$ to a relative population of singlets and triplets in a ratio of $1: 3$. The close proximity of the charge injection layers $(\sim 1 \mathrm{~nm})$ to the emissive monolayer in our devices provides a different route, which leads to the selective excitation of spin triplets.

While the architecture of our devices is significantly different to conventional OLEDs, we draw analogies with luminescence generated by the tip of a scanning tunneling microscope $\mathrm{e}^{27,28}$ (STML) for which the electrodes and photon source are also in close proximity, and, moreover, the widely accepted mechanism for molecular excitation in STML is via inelastic scattering, and there are reports of photon upconversion. ${ }^{29-31}$ Furthermore, the efficiency of our devices, typically $10^{-6}-10^{-8}$ photons per electron, is similar to that observed in STML experiments. ${ }^{27}$ The coupling of tip-induced localized plasmons to an adsorbed molecule is considered to be the most significant STML emission process; ${ }^{27}$ this is unlikely to be significant in our devices, since the plasmon energy is much smaller $^{32}$ (in the range of 100 s of $\mathrm{meV}$ ) for our FLG contacts. However, recent papers have highlighted the role of triplets in STML. Specifically, $\mathrm{T}_{1}$ emission $^{33}$ has been observed in the STML from perylene tetracarboxylic dianhydride (PTCDA), although the proposed mechanism involves a charged molecule and is not accompanied by upconversion. A triplet-mediated process has been proposed for the upconversion in the STML of metal-free phthalocyanine; ${ }^{18}$ this mechanism is similar to that discussed above, but it is not accompanied by triplet emission.

These molecular/2D hybrid heterostructures provide an alternative electronic method to control the excitation of triplets, a transition that is optically forbidden, and offer a route to fundamental studies of long-lived optical excitations and 
their applications in quantum and spin-based optoelectronics, and they are also relevant to low-voltage light-emitting devices. The device architecture has many possible variations in the choice of contact materials, molecules with higher/lower energy levels, tunnel barrier width to control the current density, as well as the scaling of the active region-either down to a single or small ensemble of molecules, or up to large-area device incorporating a complete monolayer. We also envisage the integration of molecular layers with more complex multilayer and/or in-plane supramolecular order to control the coupling of neighboring molecular emitters. These solidstate devices thus provide a new route to the investigation of the physics, chemistry, and optoelectronic applications of triplet generation, emission, and upconversion.

\section{ASSOCIATED CONTENT}

\section{S Supporting Information}

The Supporting Information is available free of charge at https://pubs.acs.org/doi/10.1021/acs.nanolett.9b03787.

Calculation of shifts of PTCDI emission energy, nonresonant shifts and triplet energy, illustrated relaxed structures, selected bond lengths, illustrated HOMO, LUMO, and TD, plot of calculated potential energy curves, details of absorption, fluorescence, and phosphorescence processes, energy decomposition data, discussion of resonant shifts, experimental methods, schematic of technique for pick-up and release, comparison of EL and PL, PL and EL spectra, additional references (PDF)

\section{AUTHOR INFORMATION}

\section{Corresponding Authors}

*E-mail: simon.svatek@upm.es. (S.A.S.)

*E-mail: peter.beton@nottingham.ac.uk. (P.H.B.)

\section{ORCID}

Simon A. Svatek: 0000-0002-8104-1888

James Kerfoot: 0000-0002-6041-4833

Elena Besley: 0000-0002-9910-7603

Peter H. Beton: 0000-0002-2120-8033

\section{Author Contributions}

${ }^{\#}$ These authors made equal contributions to this work.

\section{Notes}

The authors declare no competing financial interest.

The raw data for the AFM images and the optical and electrical measurements may be accessed through the University of Nottingham Research Data Management Repository at 10.17639/nott.7020.

\section{ACKNOWLEDGMENTS}

This work was supported by the Engineering and Physical Sciences Research Council [Grant No. EP/N033906/1] and by the Leverhulme Trust [Grant No. RPG-2016-104]. K.W. and T.T. acknowledge support from the Elemental Strategy Initiative conducted by the MEXT, Japan, and the CREST (JPMJCR15F3), JST. E.A. is grateful for the Ramón y Cajal Fellowship funded by the Spanish MINECO (RYC-201518539). Computations were performed at the High Performance Computing facility at the Univ. of Nottingham. We acknowledge the use of Athena at HPC Midlands+, which was funded by the EPSRC on Grant No. EP/P020232/1 as part of the HPC Midlands+ consortium. We thank B. Sørensen Jessen for helpful discussions.

\section{REFERENCES}

(1) Mali, K. S.; Pearce, N.; De Feyter, S.; Champness, N. R. Frontiers of Supramolecular Chemistry at Solid Surfaces. Chem. Soc. Rev. 2017, 46, 2520-2542.

(2) Macleod, J. M.; Rosei, F. Molecular Self-Assembly on Graphene. Small 2014, 10, 1038-1049.

(3) Goronzy, D. P.; Ebrahimi, M.; Rosei, F.; Arramel; Fang, Y.; De Feyter, S.; Tait, S. L.; Wang, C.; Beton, P. H.; Wee, A. T. S.; et al. Supramolecular Assemblies on Surfaces: Nanopatterning, Functionality, and Reactivity. ACS Nano 2018, 12, 7445-7481.

(4) Auwärter, W.; Écija, D.; Klappenberger, F.; Barth, J. V. Porphyrins at Interfaces. Nat. Chem. 2015, 7, 105-120.

(5) Korolkov, V. V.; Baldoni, M.; Watanabe, K.; Taniguchi, T.; Besley, E.; Beton, P. H. Supramolecular Heterostructures Formed by Sequential Epitaxial Deposition of Two-Dimensional HydrogenBonded Arrays. Nat. Chem. 2017, 9, 1191-1197.

(6) Schwarze, M.; Tress, W.; Beyer, B.; Gao, F.; Scholz, R.; Poelking, C.; Ortstein, K.; Gunther, A. A.; Kasemann, D.; Andrienko, D.; et al. Band Structure Engineering in Organic Semiconductors. Science 2016, 352, 1446-1449.

(7) Chen, W.; Qi, D. C.; Huang, H.; Gao, X.; Wee, A. T. S. OrganicOrganic Heterojunction Interfaces: Effect of Molecular Orientation. Adv. Funct. Mater. 2011, 21, 410-424.

(8) Geim, A. K.; Grigorieva, I. V. Van Der Waals Heterostructures. Nature 2013, 499, 419-425.

(9) Britnell, L.; Gorbachev, R. V.; Jalil, R.; Belle, B. D.; Schedin, F.; Mishchenko, A.; Georgiou, T.; Katsnelson, M. I.; Eaves, L.; Morozov, S. V.; et al. Field-Effect Tunneling Transistor Based on Vertical Graphene Heterostructures. Science 2012, 335, 947-950.

(10) Pizzocchero, F.; Gammelgaard, L.; Jessen, B. S.; Caridad, J. M.; Wang, L.; Hone, J.; Bøggild, P.; Booth, T. J. The Hot Pick-up Technique for Batch Assembly of van Der Waals Heterostructures. Nat. Commun. 2016, 7, 11894.

(11) Wang, L.; Meric, I.; Huang, P. Y.; Gao, Q.; Gao, Y.; Tran, H.; Taniguchi, T.; Watanabe, K.; Campos, L. M.; Muller, D. A.; et al. One-Dimensional Electrical Contact to a Two-Dimensional Material. Science 2013, 342, 614-617.

(12) Kretinin, A. V.; Cao, Y.; Tu, J. S.; Yu, G. L.; Jalil, R.; Novoselov, K. S.; Haigh, S. J.; Gholinia, A.; Mishchenko, A.; Lozada, M.; et al. Electronic Properties of Graphene Encapsulated with Different TwoDimensional Atomic Crystals. Nano Lett. 2014, 14, 3270-3276.

(13) Withers, F.; Del Pozo-Zamudio, O.; Mishchenko, A.; Rooney, A. P.; Gholinia, A.; Watanabe, K.; Taniguchi, T.; Haigh, S. J.; Geim, A. K.; Tartakovskii, A. I.; et al. Light-Emitting Diodes by Band-Structure Engineering in van Der Waals Heterostructures. Nat. Mater. 2015, 14, 301-306.

(14) Kerfoot, J.; Korolkov, V. V.; Svatek, S. A.; Alkhamisi, M.; Taniguchi, T.; Watanabe, K.; Parkinson, P. W.; Beton, P. H. TwoDimensional Diffusion of Excitons in a Perylene Diimide Monolayer Quenched by a Fullerene Heterojunction. J. Phys. Chem. C 2019, 123, 12249-12254.

(15) Kerfoot, J.; Korolkov, V. V.; Nizovtsev, A. S.; Jones, R.; Taniguchi, T.; Watanabe, K.; Lesanovsky, I.; Olmos, B.; Besley, N. A.; Besley, E.; et al. Substrate-Induced Shifts and Screening in the Fluorescence Spectra of Supramolecular Adsorbed Organic Monolayers. J. Chem. Phys. 2018, 149, No. 054701.

(16) Wewer, M.; Stienkemeier, F. Laser-Induced Fluorescence Spectroscopy of N,N'-Dimethyl 3,4,9,10-Perylene Tetracarboxylic Diimide Monomers and Oligomers Attached to Helium Nanodroplets. Phys. Chem. Chem. Phys. 2005, 7, 1171-1175.

(17) Binder, J.; Howarth, J.; Withers, F.; Molas, M. R.; Taniguchi, T.; Watanabe, K.; Faugeras, C.; Wysmolek, A.; Danovich, M.; Fal'ko, V. I.; et al. Upconverted Electroluminescence via Auger Scattering of Interlayer Excitons in van Der Waals Heterostructures. Nat. Commun. 2019, 10, 2335. 
(18) Chen, G.; Luo, Y.; Gao, H.; Jiang, J.; Yu, Y.; Zhang, L.; Zhang, Y.; Li, X.; Zhang, Z.; Dong, Z. Spin-Triplet-Mediated Up-Conversion and Crossover Behavior in Single-Molecule Electroluminescence. Phys. Rev. Lett. 2019, 122, 177401.

(19) Ford, W. E.; Kamat, P. V. Photochemistry of 3,4,9,10Perylenetetracarboxylic Dianhydride Dyes. 3. Singlet and Triplet Excited-State Properties of the Bis(2,5-Di-Tert-Butylphenyl)Imide Derivative. J. Phys. Chem. 1987, 91, 6373-6380.

(20) Uemura, T.; Furumoto, M.; Nakano, T.; Akai-Kasaya, M.; Saito, A.; Aono, M.; Kuwahara, Y. Local-Plasmon-Enhanced upConversion Fluorescence from Copper Phthalocyanine. Chem. Phys. Lett. 2007, 448, 232-236.

(21) Singh-Rachford, T. N.; Haefele, A.; Ziessel, R.; Castellano, F. N. Boron Dipyrromethene Chromophores: Next Generation Triplet Acceptors/Annihilators for Low Power Upconversion Schemes. J. Am. Chem. Soc. 2008, 130, 16164-16165.

(22) Murakami, Y. Photochemical Photon Upconverters with Ionic Liquids. Chem. Phys. Lett. 2011, 516, 56-61.

(23) Stipe, B. C.; Rezaei, M. A.; Ho, W. Single-Molecule Vibrational Spectroscopy and Microscopy. Science 1998, 280, 1732-1735.

(24) Adkins, C. J.; Phillips, W. A. Inelastic Electron Tunnelling Spectroscopy. J. Phys. C: Solid State Phys. 1985, 18, 1313-1346.

(25) Vdovin, E. E.; Mishchenko, A.; Greenaway, M. T.; Zhu, M. J.; Ghazaryan, D.; Misra, A.; Cao, Y.; Morozov, S. V.; Makarovsky, O.; Fromhold, T. M.; et al. Phonon-Assisted Resonant Tunneling of Electrons in Graphene-Boron Nitride Transistors. Phys. Rev. Lett. 2016, 116, 186603.

(26) Mitschke, U.; Bäuerle, P. The Electroluminescence of Organic Materials. J. Mater. Chem. 2000, 10, 1471-1507.

(27) Kuhnke, K.; Große, C.; Merino, P.; Kern, K. Atomic-Scale Imaging and Spectroscopy of Electroluminescence at Molecular Interfaces. Chem. Rev. 2017, 117, 5174-5222.

(28) Doppagne, B.; Chong, M. C.; Bulou, H.; Boeglin, A.; Scheurer, F.; Schull, G. Electrofluorochromism at the Single-Molecule Level. Science 2018, 361, 251-255.

(29) Dong, Z. C.; Zhang, X. L.; Gao, H. Y.; Luo, Y.; Zhang, C.; Chen, L. G.; Zhang, R.; Tao, X.; Zhang, Y.; Yang, J. L.; et al. Generation of Molecular Hot Electroluminescence by Resonant Nanocavity Plasmons. Nat. Photonics 2010, 4, 50-54.

(30) Peters, P.-J.; Xu, F.; Kaasbjerg, K.; Rastelli, G.; Belzig, W.; Berndt, R. Quantum Coherent Multielectron Processes in an Atomic Scale Contact. Phys. Rev. Lett. 2017, 119, No. 066803.

(31) Xu, F.; Holmqvist, C.; Belzig, W. Overbias Light Emission Due to Higher-Order Quantum Noise in a Tunnel Junction. Phys. Rev. Lett. 2014, 113, No. 066801.

(32) Low, T.; Avouris, P. Graphene Plasmonics for Terahertz to Mid-Infrared Applications. ACS Nano 2014, 8, 1086-1101.

(33) Kimura, K.; Miwa, K.; Imada, H.; Imai-Imada, M.; Kawahara, S.; Takeya, J.; Kawai, M.; Galperin, M.; Kim, Y. Selective Triplet Exciton Formation in a Single Molecule. Nature 2019, 570, 210-213. 